\title{
O problema que tornou Euler famoso
}

\author{
Jairo Gayo, Roy Wilhelm Probst, \\ Universidade Tecnológica Federal do Paraná, \\ 80230-901, Curitiba, PR \\ E-mail: jairogayo@yahoo.com.br, rwprobst@utfpr.edu.br.
}

\begin{abstract}
Resumo: Este trabalho apresenta o problema da Basileia, cuja resposta tornou Leonhard Euler famoso. Apresenta a prova de Euler, que por muito tempo continha uma passagem tida como incorreta, mas que após o teorema da fatoração de Weierstrass foi aceita. Aborda também outras resoluções para o problema, sua representação geométrica e uma aplicação. Discute também, por meio deste problema, a importância da história da matemática para o professor desta disciplina.
\end{abstract}

Palavras-chave: Problema da Basileia, Leonhard Euler, História da Matemática.

\section{Introdução}

Ao longo da história da matemática, foram diversos os problemas que permaneceram em aberto por anos, alguns por séculos, até que uma mente brilhante conseguisse a solução final. Atualmente são muitos os problemas que permanecem em abertos à espera de alguém que possa solucioná-los. Geralmente matemáticos que conseguem resolver um destes problemas garantem seu lugar na história da matemática.

Um dos matemáticos que alcançou notoriedade após resolver um problema em aberto foi Leonhard Euler, após encontrar a resposta para o famoso problema da Basileia. Atualmente Euler é reconhecido como um dos mais importantes da história da matemática, não só por ter resolvido tal problema, mas principalmente por ter contribuído com a matemática em diversas áreas com mais de 850 trabalhos [10], muitos de extrema importância.

Neste trabalho apresentamos o problema da Basileia (que tornou Leonhard Euler famoso), a solução apresentada por ele, algumas de suas diversas soluções ao longo da história (as aceitas atualmente) e a utilização da história da matemática como ferramenta de ensino.

\section{O problema da Basileia}

O problema que tornou Leonhard Euler famoso foi proposto inicialmente pelo matemático italiano Pietro Mengoli em 1644 [11]. Após ter consumido esforços dos maiores matemáticos do período, a solução ainda não havia surgido. Criava-se em torno deste problema um mistério semelhante ao que envolvia o último teorema de Fermat. Entre os gênios que tentaram resolvêlo estavam Gottfried Wilhelm Leibniz, John Wallis, Henry Oldenburg e os matemáticos da família Bernoulli [3].

Durante algum tempo Jakob Bernoulli atacou (sem sucesso) o problema, mas publicou algo sobre o assunto em 1689. Com a morte de Jakob, seu irmão Johann Bernoulli ocupou seu cargo de professor na Universidade da Basileia, onde teve entre seus alunos Leonhard Euler [3]. Provavelmente foi Johann que apresentou o problema à Euler, até então apenas um aluno brilhante. Após resolver o problema passou a chamar a atenção não só de seus professores na Universidade da Basileia, mas de toda a comunidade acadêmica da época.

Este problema ficou conhecido como Problema da Basileia, pois Euler nasceu e se formou nesta cidade, bem como resolveu o problema lá. Foi nela ainda que outros matemáticos de renome, como os da família Bernoulli trabalharam e tentaram resolvê-lo [11]. 
O problema proposto por Mengoli consistia em apresentar o resultado da soma dos inversos dos quadrados perfeitos, ou seja,

$$
\sum_{n=1}^{\infty} \frac{1}{n^{2}}=\frac{1}{1^{2}}+\frac{1}{2^{2}}+\frac{1}{3^{2}}+\frac{1}{4^{2}}+\ldots
$$

Antes de Euler, já se sabia que a série é convergente [7]. Basta notarmos que

$$
\begin{aligned}
\frac{1}{2 \cdot 2}+\frac{1}{3 \cdot 3}+\cdots+\frac{1}{k \cdot k} & <\frac{1}{1 \cdot 2}+\frac{1}{2 \cdot 3}+\cdots+\frac{1}{(k-1) k} \\
& =\frac{1}{1}-\frac{1}{2}+\frac{1}{2}-\frac{1}{3}+\cdots+\frac{1}{k-1}-\frac{1}{k} \\
& =1-\frac{1}{k} .
\end{aligned}
$$

Quando $k$ tende ao infinito, temos

$$
\lim _{k \rightarrow \infty}\left[1-\frac{1}{k}\right]=1
$$

$\log 0$

e portanto

$$
\frac{1}{2^{2}}+\frac{1}{3^{2}}+\cdots<1
$$

ou seja

$$
\frac{1}{1^{2}}+\frac{1}{2^{2}}+\frac{1}{3^{2}}+\cdots<\frac{1}{1^{2}}+1
$$

$$
\sum_{n=1}^{\infty} \frac{1}{n^{2}}<2 .
$$

Portanto, esta série é crescente e limitada superiormente, logo é convergente.

Somente em 1735, mais de 90 anos após o lançamento da questão, Leonhard Euler apresentou uma solução aceita na época que surpreendeu a todos devido a sua originalidade [11].

\subsection{A Prova de Euler}

Na solução apresentada por Euler [4], inicialmente tomamos a série de Maclaurin da função seno

$$
\operatorname{sen} x=x-\frac{x^{3}}{3 !}+\frac{x^{5}}{5 !}-\frac{x^{7}}{7 !}+\cdots
$$

dividimos ambos os lados desta equação por $x$

$$
\frac{\operatorname{sen} x}{x}=1-\frac{x^{2}}{3 !}+\frac{x^{4}}{5 !}-\frac{x^{6}}{7 !}+\cdots
$$

e substituimos $x$ por $\sqrt{x}$

$$
\frac{\operatorname{sen} \sqrt{x}}{\sqrt{x}}=1-\frac{x}{3 !}+\frac{x^{2}}{5 !}-\frac{x^{3}}{7 !}+\cdots
$$

Os zeros da função

$$
f(x)=\frac{\operatorname{sen} \sqrt{x}}{\sqrt{x}}
$$

são $\pi^{2}, 4 \pi^{2}, 9 \pi^{2}, 16 \pi^{2}, \ldots$ 
Já era conhecido na época de Euler que todo polinômio

$$
p(x)=x^{n}+a_{n-1} x^{n-1}+\ldots+a_{2} x^{2}+a_{1} x+a_{0}
$$

com raízes $r_{1}, r_{2}, \ldots, r_{n}$ pode ser escrito como um produto envolvendo suas raízes

$$
\left(x-r_{1}\right)\left(x-r_{2}\right)\left(x-r_{3}\right) \ldots\left(x-r_{n}\right)=x^{n}+a_{n-1} x^{n-1}+\ldots+a_{2} x^{2}+a_{1} x+a_{0}
$$

de onde obtemos

$$
\begin{aligned}
& a_{0}=(-1)^{n} r_{1} r_{2} \ldots r_{n} \\
& a_{1}=(-1)^{n-1} r_{2} r_{3} \ldots r_{n}+(-1)^{n-1} r_{1} r_{3} \ldots r_{n}+\ldots+(-1)^{n-1} r_{1} r_{2} \ldots r_{n-1}
\end{aligned}
$$

e dividindo $a_{1}$ por $a_{0}$, obtemos

$$
-\frac{a_{1}}{a_{0}}=\frac{1}{r_{1}}+\frac{1}{r_{2}}+\cdots+\frac{1}{r_{n}} .
$$

Assumindo que esta propriedade dos polinômios continua valendo para séries de potências e aplicando à função $f(x)$, concluímos que

$$
\frac{1}{6}=\frac{1}{\pi^{2}}+\frac{1}{4 \pi^{2}}+\frac{1}{9 \pi^{2}}+\frac{1}{16 \pi^{2}}+\cdots
$$

e multiplicando ambos os lados desta equação por $\pi^{2}$ obtemos

$$
\frac{\pi^{2}}{6}=\frac{1}{1^{2}}+\frac{1}{2^{2}}+\frac{1}{3^{2}}+\frac{1}{4^{2}}+\cdots
$$

O resultado obtido por Euler era surpreendente por envolver $\pi$ na resposta de uma série que aparentemente não estava relacionada com circunferências ou trigonometria. O próprio Euler, devido a este fato, relutou em apresentar a prova por um breve tempo [11].

No entanto, apesar da resposta estar correta e da belíssima demonstração, existiam alguns passos que não estavam bem explicados.

\subsection{A invalidade da prova de Euler}

O problema da prova de Euler é a utilização da propriedade dos polinômios (1) em uma séries de potências, o que nem sempre é válido. Basta observarmos a identidade

$$
\frac{1}{1-x}=1+x+x^{2}+x^{3}+\ldots
$$

válida para todo $x \in(-1,1)$, e a função

$$
g(x)=\frac{1}{2}-\frac{1}{1-x} .
$$

É fácil perceber que o único zero da função $g(x)$ é $r_{1}=-1$. Reescrevendo esta função temos

$$
g(x)=-\frac{1}{2}-x-x^{2}-x^{3}-\ldots
$$

Note que a região de convergência da série é diferente do domínio da função e que $r_{1}$ não pertence à região de convergência da série. Mas, assumindo que a propriedade (1) continua valendo para esta série, temos

$$
-\frac{-1}{-1 / 2}=\frac{1}{-1}
$$

ou seja $1=2$, o que deixava a prova de Euler inválida. 
Mas em 1885 Weierstrass demonstrou o que ficou conhecido como teorema da fatoração de Weierstrass [8]: Toda função analítica (aquelas que podem ser representadas por uma série de potências) pode ser expressa por um produto envolvendo suas raízes.

Realmente, a função

$$
f(x)=\frac{\operatorname{sen} \sqrt{x}}{\sqrt{x}}
$$

é uma função analítica e pode ser representada por uma série de potências em todo seu domínio, passo justificado pelo teorema da fatoração de Weierstrass. O problema é que a resolução de Euler é anterior a este teorema. A resolução de Euler é de 1735, ou seja, para uma das passagens de sua resolução não havia justificativa por aproximadamente 150 anos. Foi neste período que outros matemáticos, ao mesmo tempo em que aplaudiam o resultado obtido, chamavam a atenção para falta de rigor desta passagem. Muitos matemáticos, também brilhantes, conseguiram soluções contendo apenas passagens válidas para o problema da Basileia. O trabalho destes, com certeza de grande valia para a matemática, foi de certa forma facilitado, uma vez que já conheciam o resultado numérico do problema.

O teorema da fatoração de Weierstrass dificilmente é apresentado em livros de cálculo para iniciantes devido sua complexidade. Apresentar a prova de Euler sem mencionar o teorema de Weierstrass incorre em falta de rigor matemático, invalidando a prova e tornando-a inválida neste nível.

\section{Provas rigorosas do problema da Basileia}

Após Euler chegar a resposta do problema da Basileia muitos outros matemáticos também apresentaram suas resoluções [6]. Dentre as provas para o problema da Basileia estão algumas bem elementares e outras um tanto quanto complexas. Nesta seção serão apresentados os resumos de algumas em diferentes níveis [5].

\subsection{A prova de Cauchy}

A primeira resolução conhecida é devida à Augustin-Louis Cauchy . Esta prova se destaca por utilizar apenas conhecimentos de matemática elementar.

Apesar de utilizar o teorema do confronto (um resultado mais avançado, porém intuitivo), nas demais passagens a prova de Cauchy utiliza apenas conhecimentos de polinômios, trigonometria e números complexos, conteúdos do currículo nacional do ensino médio. Sendo assim, esta é uma das provas válidas mais simples para o problema.

\subsection{A prova de Fourier}

Esta resolução utiliza a série de Fourier de $f(x)=x^{2}$ no intervalo $(-\pi, \pi)$ :

$$
f(x)=\frac{\pi^{2}}{3}+4 \sum_{n=1}^{\infty} \frac{(-1)^{n} \cos (n x)}{n^{2}}
$$

Para $x=\pi$ obtemos a solução do problema da Basileia.

\subsection{A prova de Apostol}

A resolução mais recente do problema é atribuída a Apostol [2] e envolve o cálculo da integral

$$
\int_{0}^{1} \int_{0}^{1} \frac{1}{1-x y} d x d y
$$

A prova de Apostol é do século XX, a de Fourier é do século XIX e do século XVIII temos as provas de Cauchy e Euler. A prova de cada período traz características da matemática desenvolvida até seu tempo, mostrando que um assunto dentro da matemática nunca está finalizado. 


\section{Curiosidades do problema da Basileia}

Para finalizar este trabalho vamos apresentar a representação geométrica e uma aplicação da série. Estas duas curiosidades mostram que, apesar de antiga, esta série ainda é um tema atual.

\subsection{Representação geométrica}

O fato de estarmos trabalhando com uma série de quadrados, nos faz pensar inicialmente em teorema de Pitágoras [9]. Tomamos então as duas primeiras parcelas da soma e montamos um triângulo retângulo de vértices nos pontos $A, P_{1}$ e $P_{2}$, de forma que $\overline{A P_{1}}=1, \overline{P_{1} P_{2}}=1 / 2$ e que o ângulo em $p_{1}$ seja reto conforme figura 1 . Para facilitar a representação fazemos $r_{1}=\overline{A P_{1}}$ e $r_{2}=\overline{A P_{2}}$. Com o teorema de Pitágoras obtemos

$$
r_{2}^{2}=1^{2}+\left(\frac{1}{2}\right)^{2}=1+\frac{1}{2^{2}}
$$

Marcamos agora o ponto $P_{3}$ de forma que $\overline{P_{2} P_{3}}=1 / 3$ e que o ângulo $\widehat{A P_{2} P_{3}}$ seja reto. Assim o triângulo $A P_{2} P_{3}$ é retângulo e considerando $\overline{A P_{3}}=r_{3}$ temos

$$
r_{3}^{2}=r_{2}^{2}+\left(\frac{1}{3}\right)^{2}=1+\frac{1}{2^{2}}+\frac{1}{3^{2}}
$$

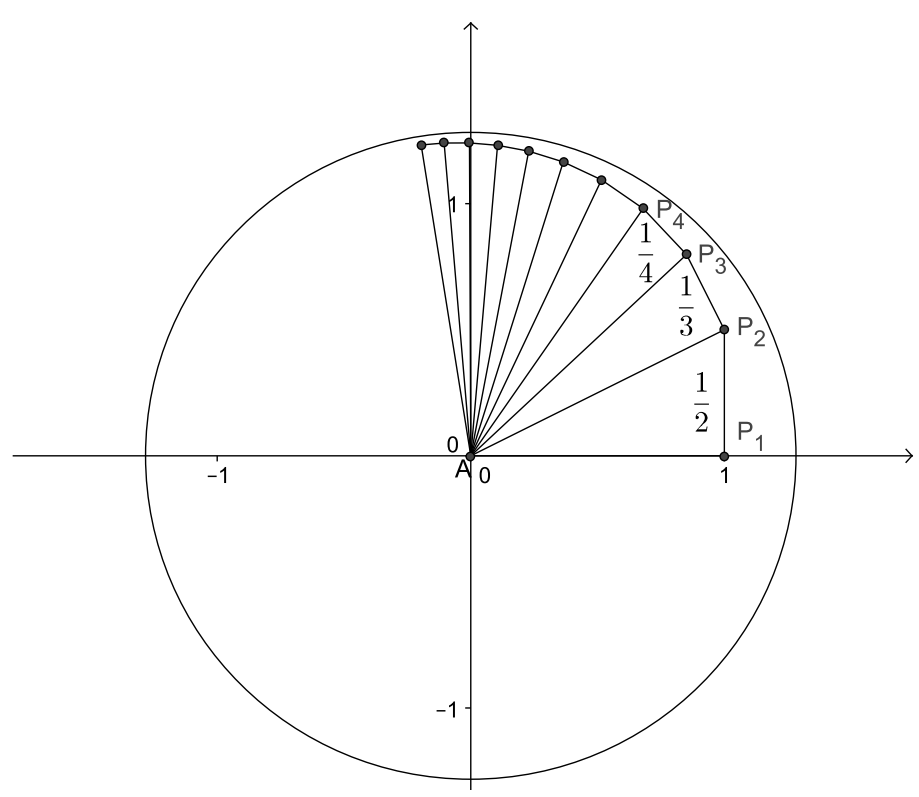

Figura 1: O teorema de Pitágoras e o problema da Basileia

Continuando a marcar pontos desta forma, ao marcarmos o ponto $P_{n}$, conforme a figura 1 temos um segmento $r_{n}$ onde vale a seguinte igualdade

$$
r_{n}^{2}=1+\frac{1}{2^{2}}+\frac{1}{3^{2}}+\ldots+\frac{1}{n^{2}}
$$

Assim, pelo problema da Basileia é verdade que

$$
r_{n}^{2}<\frac{\pi^{2}}{6}
$$

ou ainda

$$
r_{n}<\frac{\pi}{\sqrt{6}} .
$$


Desta forma, continuando com a sequência, teremos a certeza que todos os pontos $P_{n}$ estarão contidos dentro do círculo de raio $\pi / \sqrt{6}$ e centro em $A$.

Percebemos que os segmentos $\overline{A P_{1}}, \overline{P_{1} P_{2}}, \overline{P_{2} P_{3}}, \ldots, \overline{P_{n} P_{n+1}}, \ldots$ formam uma espiral. Realmente, somando a medida de cada segmento temos a série harmônica

$$
1+\frac{1}{2}+\frac{1}{3}+\frac{1}{4}+\ldots
$$

que pelo fato de ser divergente gera uma espiral infinita. Esta espiral por sua vez, se afasta cada vez mais do ponto $A$, se aproximando cada vez mais da circunferência sem ultrapassá-la.

\subsection{Probabilidade}

Agora vamos mostrar uma aplicação do problema da Basileia. Esta série será utilizada para calcular a probabilidade de dois números serem primos entre si [1]. Mais precisamente, calcular a probabilidade de dois números menores que $n$ serem primos entre si, quando $n$ tende ao infinito.

Seja $g$ o máximo divisor comum de dois inteiros positivos $a$ e $b$, isto é $g=(a, b)$ e seja $p$ a probabilidade de que $g=1$. Primeiro vamos mostrar que a probabilidade de que $g=n$ para $n=1,2,3, \ldots$ é $p / n^{2}$.

A probabilidade de que $n$ divide tanto $a$ quanto $b$ é $1 / n^{2}$. Para que, além de dividir $a$ e $b$, $n$ seja o máximo divisor comum entre $a$ e $b$, é necessário que $(a / n, b / n)=1$, o que ocorre com probabilidade $p$. Portanto a probabilidade de que $g=n$ é $p / n^{2}$.

Como a soma das probabilidades de que $g=n$ para $n=1,2,3, \ldots$ deve ser igual a 1 , temos

$$
\sum_{n=1}^{\infty} \frac{p}{n^{2}}=1 .
$$

Isolando $p$, obtemos

$$
p=\frac{1}{\sum_{n=1}^{\infty} \frac{1}{n^{2}}}=\frac{1}{\left(\frac{\pi^{2}}{6}\right)}=\frac{6}{\pi^{2}} .
$$

Como uma consequência interessante deste resultado, a probabilidade de uma fração gerada aleatoriamente ser irredutível é $6 / \pi^{2} \cong 60,8 \%$ [7].

\section{Conclusões}

A ideia deste trabalho é mostrar, através do problema que tornou Leonhard Euler famoso, a interessante ferramenta didática que é a história da matemática para os professores desta disciplina. O objetivo não é contar a história da matemática por meio de uma maçante biografia, ou por meio de fatos pitorescos ocorridos com matemáticos, mas sim contar esta história mostrando como um assunto surge, se desenvolve e ganha importância ao longo do tempo.

Pietro Mengoli era professor universitário de matemática na Itália em uma época em que o Cálculo ainda não havia sido inventado por Newton e Leibniz, porém já se estudava a convergência de séries. Provavelmente foi estudando este assunto que Mengoli teve a ideia de sugerir o problema. Não há evidências de que ele propôs esta questão com o objetivo de resolver um problema aplicado a uma situação real. Este fato mostra que nem todo conhecimento matemático surge de uma necessidade prática da humanidade.

Este problema circulou entre os melhores matemáticos da época, sua convergência foi provada de uma forma tão simples que hoje em dia um aluno do ensino médio compreenderia sem muito esforço. No entanto, na busca do valor exato da soma infinita, muitos matemáticos se desdobraram e muitas ideias surgiram, uma delas foi o teste da integral para séries convergentes. Isto mostra mais uma curiosidade da matemática por meio de sua história: ao se procurar uma coisa, encontra-se outra. 
Então chegou a vez de Euler, ele produziu tanto que alcançou notoriedade por muitas outras contribuições à matemática, mas foi resolvendo o problema da Basileia que se destacou pela primeira vez. Sua prova tinha um equívoco, o que não tirava sua beleza haja visto que o resultado estava certo. Neste ponto a história nos mostra que o raciocínio, a criatividade e o conhecimento são tão importantes na matemática quanto seu rigor e formalismo.

Na verdade o equívoco ocorre na utilização de uma propriedade dos polinômios em uma série de potências, que como vimos, caso Euler quisesse justificá-la corretamente hoje seria perfeitamente possível com o teorema de Weierstrass. Aqui percebemos que no desenvolvimento da matemática, a intuição e o pensamento vem a frente do formalismo e dos teoremas. Percebemos ainda que a matemática não se desenvolve linearmente, tal como está em um livro didático: sua evolução é dinâmica, partes que compõem um mesmo tema surgiram e se desenvolveram em tempos, lugares e com finalidades diferentes.

Enquanto não surgia o teorema de Weierstrass, muitos matemáticos se mobilizaram para apresentarem suas provas para o problema. Diversas provas surgiram, todas com suas peculiaridades, isso nos mostra que apesar da matemática ser exata, um problema pode ser resolvido de diversas maneiras, basta observarmos as provas apresentadas no texto, que com certeza não são as únicas [6].

A representação geométrica da série mostra que as áreas da matemática se relacionam. Estas relações também podem ser percebidas na aplicação encontrada para a série. Perceba que nosso problema está ligado ao teorema de Pitágoras, limites, circunferências, probabilidade, frações e divisibilidade. Isto nos leva a refletir que a aplicação de um conhecimento pode vir através da relação entre conteúdos.

Estas considerações mostram como a história da matemática pode ser utilizada como uma poderosa ferramenta de motivação e uma possibilidade de metodologia de ensino.

\section{Referências}

[1] A.D. Abrams e M.J. Paris, The Probability That $(a, b)=1$, College Mathematics Journal, 23 (1992).

[2] T.M. Apostol, A proof that Euler missed: evaluating $\zeta(2)$ the Easy Way, The Mathematical Intelligencer, 5 (1983).

[3] C.B. Boyer, "História da Matemática", Editora Edgard Blücher, São Paulo, 2003.

[4] L. Euler, De Summis Serierum Reciprocarum, Commentarii Academiae Scientiarum Petropolitanae, Opera Omnia, (1740).

[5] J. Gayo, "O problema que tornou Euler Famoso", Dissertação de Mestrado, PROFMATUTFPR, 2013.

[6] D.P. Giesy, Still another elementary proof that $\sum 1 / k^{2}=\pi^{2} / 6$, Mathematics Magazine, 45 (1972).

[7] D. Kalman, Six Ways to Sum a Series, The College Mathematics Journal, 24 (1993).

[8] K. Knopp, "Theory of Functions, Part II", Dover, New York, 1996.

[9] E. Maor, "The Pythagorean Theorem: A 4,000-Year History", Princeton University Press, Princeton, 2007.

[10] J.C. Simmons, "Os 100 maiores cientistas da História: Uma classificação dos cientistas mais influentes do passado e do presente", DIFEL, Rio de Janeiro, 2002.

[11] G.G. Szpiro, "A vida secreta dos números", DIFEL, Rio de Janeiro, 2008. 